\section{The influence of age and presentation order upon children's free recall and learning to learn*}

\author{
EMOGENE K. YOSHIMURA, BARBARA E. MOELY, and S. I. SHAPIRO \\ University of Hawaii, Honolulu, Hawaii 96822
}

Free recall and clustering performance were studied in children of ages 4 and 9-10 years. Nine different lists were presented, each for three trials, with three lists presented per day. The lists were composed of conceptually related or unrelated items. Each list type was presented consistently with all the items randomized, with conceptually related items grouped together, or with unrelated items arbitrarily grouped together. Performance was generally higher for the older children, but no evidence was found in either age group of learning-to-learn effects of recall or category clustering.

This study was designed to assess the influence of age level, presentation method, and learning to learn upon children's free recall and category clustering performance. A previous report by Moely \& Shapiro (1971) indicated that with children of ages 3 , 4,5 , and 6-7 years, learning to learn of recall and clustering occurred over a series of sessions but was dependent upon consistent block presentation of pairs of items rather than upon conceptual relations per se. It is possible, however, that the oldest children may have been in a transition stage, after which the cognitive-memorial abilities involved in learning to learn of conceptual relations in a free recall task might emerge. Thus, for example, Moely, Olson, Halwes, \& Flavell (1969) have provided evidence that verbal rehearsal and deliberate self-testing in a recall task begin to emerge in children somewhat older than those tested by Moely \& Shapiro (1971). The present study, therefore, was conceived as a replication and extension of the study by Moely \& Shapiro (1971). Children tested were older than those in the earlier investigation, and the number of training sessions was increased from six to nine sessions. Thus, each $\mathrm{S}$ was

* This research is based upon a Master's thesis submitted by the first author to the University of Hawaij in which additional details may be consulted (Yoshimura, 1970). Support was provided by a grant to the second author from the University of Hawaii Research Council. The authors would like to thank the administrators and teachers of Playmate Kindergarten and Day Care Center, Our Redeemer Lutheran Church, and the University of Hawaii Laboratory School, all in Honolulu, Hawaii. Special thanks are due Miyo Hee Ko, Phillip Roth, and Ronald Mitchell for their generous cooperation in making this study possible. Helpful comments were contributed by Richard Dubanoski, and computer time was made available by the University of Hawaii Statistical and Computing Center. presented with nine different lists for three trials per list, with three lists presented per day. In addition, triads of conceptually related items, rather than pairs, were employed so that the discovery and utilization of conceptual relations might be enhanced. The intent of instituting the modifications outlined was to determine whether or not learning-to-learn effects could be established, based upon conceptual relations per se. As in the earlier study, lists were presented of conceptually related or unrelated items and with either block or random presentation of the items.

\section{METHOD}

The Ss were 40 children of ages $4: 0$ to $4: 11$ years $(M=4: 4)$ and 40 children of ages $9: 1$ to $10: 10$ years $(M=10: 3)$. An additional three Ss were dropped for procedural problems. The Ss were obtained randomly from one preschool and two private elementary schools in Honolulu, Hawaii. The majority of the Ss came from middle-income families and represented a mixture of ethnic backgrounds. The Ss selected had to be able to verbalize reasonably well according to teachers' reports. The Ss were assigned randomly to one of four treatment conditions, with the restriction that five $S s$ of each sex at each age level were assigned to each of the conditions. The female $E$ was an experienced $E$ of Japanese ancestry.

The stimuli were 108 specially produced line drawings of common objects preestablished to be familiar to children. The items were approximately $2 \times 2$ in. and mounted on $4 \times 6$ in. laminated cards. The items represented 36 conceptual categories, each consisting of three items preestablished to be conceptually related to one another. The 108 items were grouped into nine lists of 12 items each ${ }^{1}$ such as to minimize relationships between the items of different triads within a list. A
2 by 2 by 2 by 3 by 3 design was employed, in which comparisons were made between 2 age groups (4- vs 9 - to 10-year-olds), 2 types of lists (expefimental vs control), 2 presentation conditions (block vs random), 3 days of testing, and 3 sessions within each day. Each $\mathbf{S}$ was tested with all 108 items, but under one of four conditions defined by the nature and order of the list items presented. In the experimental blocked condition, the items were arranged in each list so that the members of conceptually related triads always followed one another in presentation. The triad blocks themselves were randomized on each trial so that no triad appeared more than once in each block serial position over trials. Within a triad, the three items were randomly varied from trial to trial. For the experimental random condition, the items comprising the lists were the same as for the experimental blocked condition, except that the items were presented randomly on each trial, with the restriction that no two members of the same category occurred in succession, and there were no repetitions of any two unrelated items across trials. In the two control list conditions, the 108 items were rearranged into nine lists each consisting of 12 unrelated items. To minimize interference effects, two intervening lists were presented before a list was given in which members of a category from the first list appeared a second time. For the control block condition, the items in each list were arranged so that arbitrarily designated triads of items were consistently presented contiguously on each trial. The randomization procedures were the same as for the experimental blocked condition. In the control random condition, the same lists were used as for the control blocked condition, except that the randomization procedures followed were the ones used for the category random condition.

The Ss were tested individually in a relatively quiet distraction-free room in the school. A short practice list of three unrelated items (different from the experimental stimuli) was given at the first session to familiarize the $\mathrm{Ss}$ with the procedure and to minimize warm-up effects. For all sessions, the $\mathrm{S}$ was asked to look at each drawing, say its narie, and, after all the items of a list wede presented, attempt to recall the namies of all the stimuli in any order. For every item correctly recalled; the $E$ verbally reinforced the child and gave him a token to be exchanged later for a prize. Various verbal prompts were used to encourage the $\mathrm{S}^{-}$to recall as many items as 
possible. The recall period was terminated when the $E$ judged the $S$ could not recall any more items. The stimuli were presented one at a time at a 3 -sec rate. The Ss rarely failed to label an item, but when this did occur, the $E$ supplied the appropriate name. Three alternating presentation and recall periods were given for each list, and oral recall was obtained immediately following each presentation. A tape recorder was used to check E's written record of the responses. Each $S$ was exposed to three different lists (sessions) per day for 3 consecutive school days. Each session of three trials lasted about $10 \mathrm{~min}$. The within-day sessions were separated by a $1-h$ interval which the $S$ spent back in his classroom. The lists were presented to Ss within each age level and within each treatment condition such that equal numbers of Ss received each list in each of the nine recall sessions given.

RESULTS AND DISCUSSION

The data analyzed were the number of items recalled, category clustering, and intrusion responses (i.e., items not on the list). Duplicate (i.e., items repeated more than once) and intrusion responses were omitted in calculating recall and clustering performance. Recall and clustering performance were both analyzed by means of an analysis of variance comprised of the between-S factors of age (younger vs older), list type (experimental vs control), presentation method (block vs random), and the within-S factors of days (three levels) and sessions (three levels). The Duncan multiple range test was employed for the individual comparisons. Only seven Ss produced intrusion responses, and the number of such Ss was not significantly associated with any particular age level, list type, or presentation method. The minimal number of intrusions suggests that proactive interference was not an important influence on performance, even for the control conditions where conceptually related members appeared in different lists. Duplicate responses were so few that no further analyses were undertaken. Figure 1 presents mean recall performance averaged over trials and sessions within a day for each age group and type of list. The analysis of variance yielded significant main effects of age $[F(1,72)=344.70, \quad p<.001]$, list type $[F(1,72)=24.69, \quad p<.001]$, sessions $[F(2,144)=5.08, p<.01]$, and significant interactions of Age by List Type by Days $[F(2,144)=7.30$, $\mathrm{p}<.001]$, and List Type by Sessions $[F(2,144)=4.14, p<.05]$. These results reflect that recall was higher for the older than for the younger Ss, higher for categorized than for control

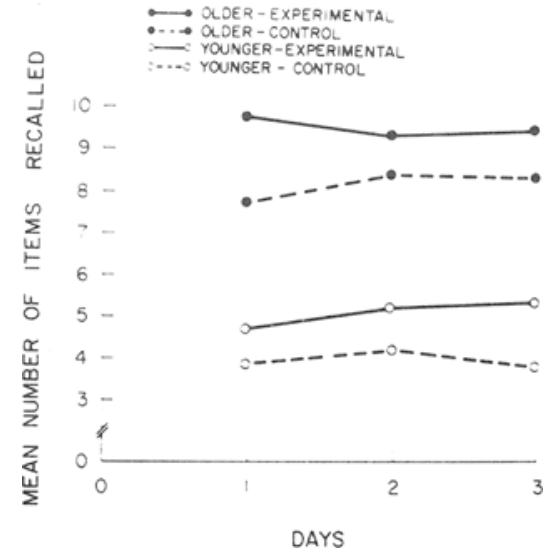

Fig. 1. Mean recall performance over 3 days as a function of age group and type of list.

lists, recall increased from Session 1 to Session 2 for the older Ss but declined from Session 2 to Session 3, and, as Fig. 1 indicates, there is little consistency across days within groups. Thus, recall did not systematically increase across days or sessions. Figure 2 presents mean clustering performance averaged over trials and sessions within a day for each age group and type of list. The clustering measure employed was Bousfield \& Bousfield's (1966) observed minus expected stimulus category repetitions, $O(S C R)-E(S C R)$. The analysis of variance of clustering scores yielded significant main effects of age $[F(1,72)=38.19, p<.001]$, list type $[F(1,72)=75.39, \quad p<.001], \quad$ and presentation method $[F(1,72)=75.39, p<.001]$, and significant interactions of Age by List Type $[F(1,72)=9.99, p<.05]$, Age by List Type by Presentation Method $[F(1,72)=16.34, p<.001]$, and List Type by Presentation Method $[F(1,72)=21.27, \quad p<.001] . \quad$ These results reflect that clustering was greater for the older than for the

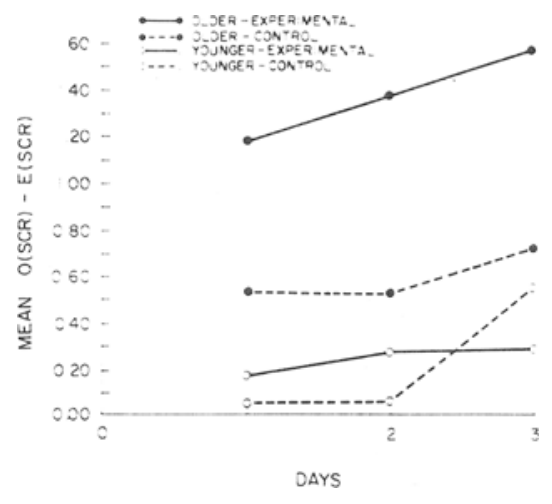

Fig. 2. Mean clustering performance over 3 days as a function of age group and type of list. younger children, and clustering was greater for the older children given the blocked experimental lists than for any of the other seven groups $(\alpha=.01)$. However, the amount of clustering did not increase significantly across days or sessions within a day, although there does appear to be a consistent increase across days for the older Ss given the blocked experimental lists.

The results provide no evidence of learning to learn either of free recall or of clustering performance, even though the children tested were older than the Ss tested by Moely \& Shapiro (1971) and despite the increased number of training sessions. In other respects, the primary results of the two studies are in agreement: recall and clustering performance improve with age, and performance with categorized lists is superior to uncategorized lists, at least with older children and blocked presentation. While neither study provides evidence of learning to learn based on conceptual relations per se, Moely \& Shapiro (1971) did find learning-to-learn effects based only upon consistent block presentation of pairs of items whether or not they were related. It may be that with consistent blocked presentation of pairs of items, the children learn to improve their performance of paired-associates learning by anticipating the second member of a blocked pair of words after being presented with the first member. In the present study, such behavior is less likely, since the blocked categories each consisted of three items.

It should be noted that there are several discrepancies in the present results between the amount of recall achieved compared to the level of category clustering. Thus, the older children consistently recalled more of the randomly presented categorized lists than the randomly presented control lists, although there were no differences in clustering performance for the two conditions, and within the younger children's group, recall for the blocked and randomly presented categorized lists was consistently higher than for the respective control lists, although there were no parallel differences in clustering performance. Such discrepancies between the amount of recall and category clustering have been also noted by Puff (1970) and have been observed with subjective organization (Shapiro \& Bell, 1970), as well as category clustering. These discrepancies call attention to the need for examining more closely current conceptualizations of the role of organization in human memory. Thus, with respect to the absence of 
learning-to-learn effects in children based on conceptual relations, it is difficult to decide whether such cognitive-memorial abilities actually do not exist for the age levels tested or whether, in the situations tested heretofore, there may be a production deficiency (e.g., Flavell, Beach, \& Chinsky, 1966), particularly if improvements in recall are not necessarily dependent upon clustering abilities.

\section{REFERENCES}

BOUSFIELD, A. K., \& BOUSFIELD, W, A Measurement of clustering and of sequential constancies in repeated free recall. Psychological Reports, 1966, 19, 935-942.

FlavelL, J. H., BEACH, D. H., \& CHINSKY, J. M. Spontaneous verbal rehearsal in a memory task as a function of age. Child Development, 1966, 37. 283-299.

MOELY, B. E., OLSON, F. A., HALWES, T. G., \& FLAVELL, J. H. Production deficiency in young children's clustered recall. Developmental Psychology, 1969 , 1, 26-34.

MOELY, B. E., \& SHAPIRO, S. I. Free recall and clustering at four age levels: Effects of learning to learn and presentation method. Developmental Psy chology, 1971, 3, in press.
PUFF, C. R. Role of clustering in free recall. Journal of Experimental Psychology, $1970,86,384-386$.

SHAPIRO, S. I., \& BELL, J. A. Subjective organization and free recall: Performance of high, moderate, and low organizers. Psychonomic Science, 1970, 21, 71-73.

YOSHIMUR A, E. K. The role of age, list type, presentation order and practice upon children's free recall learning. Unpublished Master's thesis, University of Hawai, 1970.

\section{NOTE}

1. The triads comprising the catategorized lists were: List 1 -dog, cat, cow; hand, foot, ear: bed, table, chair: drum, guitar, horn List 2-elephant, giraffe, lion: jacket, skirt, dress; ball, wagon, blocks; shovel, hammer, ladder; List 3-sun, moon, star; car, train, truck: girl, boy, lady; knife, fork, spoon List 4-soap, towel, toothbrush; house, barn, castle; flower, tree, grass; pen, pencil, crayons: List 5-butterfly, ant, bee: corn, potato, carrot: book, newspaper, sign: plate, bowl, cup; List 6-apple, pear, orange: broom, vacuum cleaner, rake: window stairs, door: shoe, sock, sandal List 7-basket, pail, bottle: clock, lamp, fan: pie, candy bar, ice cream; mountain. ocean city: List 8-goose, owl, eagle: fish, whale seal: mirror, comb, brush; padlock, key, chain: List 9-swing, seesaw, slide; ship, raft, sailboat: ring, wristwatch, earring; stove, refrigerator, sink.

\title{
Covert oral behavior during conversational and visual dreams*
}

\author{
F. J. McGUIGAN and ROBERT G. TANNER \\ Hollins College, Roanoke, Va. 24020
}

Previous findings of heightened covert oral behavior during linguistic activities suggested that increases in covert oral behavior might also occur during conversational dreams. It was found that covert oral behavior (lip and chin electromyograms) was significantly higher during rapid eye movement (REM) periods in which there were conversational dreams than during nonrapid eye movement (NREM) periods. On the other hand, REM periods for the visual dreams showed only minor and nonsignificant changes in covert oral behavior, relative to the NREM periods. Little change occurred for neck responses, suggesting that behavioral changes were localized in the speech region. These findings are thus consistent with those obtained from waking Ss-covert oral behavior may serve a linguistic function during dreams too.

Heightened covert oral behavior, as measured by electromyograms (EMG) from speech muscles, occurs during the performance of a wide variety of linguistic activities (auditory hallucinations, silent reading, writing, thinking, etc.), indicating that the covert oral response may function to

* The project reported herein was performed pursuant to a grant from the U.S. Office of Education, Department of Health. Education, and Welfare. The opinions expressed herein, however, do not necessarily reflect the position or policy of the U.S. Office of Education, and no official endorsement by the U.S. Office of Education should be inferred. facilitate internal information processing (McGuigan, 1970). An extension of these findings suggests that covert oral behavior should also be apparent during dreams which involve language. It was, therefore, predicted that there would be a noticeable increase in amplitude of covert oral behavior during conversational dreams but not visual (nonlinguistic) dreams.

\section{METHOD}

The Ss were four female undergraduates at Hollins College. Each slept in the laboratory for 4 consecutive nights and was paid $\$ 5.00$ a night. The $\mathbf{S}$ and apparatus rooms contained effective shielding for extraneous signals. EMGs were continuously recorded during sleep from the chin, lips, and neck, as were frontal electroencephalograms (EEG), and eye movements from the external canthi, all recorded on a seven-track data tape recorder. The signals were visually monitored by oscilloscopes throughout each night, and $\mathbf{S}$ was awakened after each rapid eye movement (REM) period. At that time S's dream report was recorded on an audio tape recorder, and $S$ gave a clarity rating of the dream using the method of Roffwarg, Dement, \& Muzio (1962). Those dreams that received S's highest clarity rating (viz, 3 ) were later classified for type of content; for this, three independent judges used a 5-point scale that ranged from "primarily visual content" to "primarily conversational content." Dreams judged by all three raters to be "primarily visual" or "mostly visual" were classified as "visual dreams," while those dreams which were unanimously judged to be "primarily conversational" or "mostly conversational" were classified as "conversational dreams." Recorded data were analyzed for these REM periods; "nonrapid eye movement" (NREM) comparison data were selected for an equal period of time from a point terminating $5 \mathrm{~min}$ prior to the onset of each REM period.

\section{RESULTS}

A total of 25 dreams received the high clarity rating. Thirteen of these met the independent rater criteria: eight were classified as visual and five as conversational dreams. All psychophysiological signals were integrated, digitized, and amplitudes were printed out for each REM period and for each preceding NREM period (for details, see McGuigan, 1967). Mean amplitudes for each REM and corresponding NREM period were then computed for each measure, and the latter was subtracted from the former. A mean response increase from the NREM to the REM periods was then computed for each S's visual and conversational dreams. Group means of the NREM and REM periods and their differences are presented in Table 1. It can be seen that both of the covert oral measures significantly increased from the NREM to the REM periods during conversational dreams $(A=.282$ and $A=.307$ for lip and chin EMG, respectively). In contrast, during visual dreams the differences for lip and chin EMG were minor and nonsignificant. The increases in lip and chin EMG during conversational dreams are noticeably larger than during visual dreams, but the differences in this case only approach 